\title{
A Prospective for the Potential Effect of Local Anesthetics on Stem-Like Cells in Colon Cancer
}

\author{
Hengrui Liu* \\ Department of Biochemistry, University of Cambridge, Cambridge, UK
}

*Corresponding author: Hengrui Liu, Department of Biochemistry, University of Cambridge, Cambridge CB2 1QW, UK; Department

of Anesthesiology, Health Science Center, Stony Brook University, Stony Brook, NY 11794, USA

\begin{abstract}
ARTICLE INFO
Received: 幽 January 27, 2020

Published: 幽 January 31, 2020

Citation: Hengrui Liu. A Prospective for the Potential Effect of Local Anesthetics on Stem-Like Cells in Colon Cancers. Biomed J Sci \& Tech Res 25(2)-2020. BJSTR. MS.ID.004166.
\end{abstract}

Keywords: colon cancer; local anesthetics; cancer stem cells

\begin{abstract}
Colorectal cancer is the third most prevalent cancer and the second most frequent cause of cancer-related death in the world. Surgical resection of the primary tumor is the central aspect of the current multiple modes of treatment and has been associated with better prognosis. The process of surgery, including anesthetic regimens, has increasingly been recognized to affect colon cancer recurrence and metastasis. Both retrospective clinical studies and laboratory studies have reported that colon cancer cells are inhibited by some local anesthetics. However, the application of local anesthetics in colon cancer treatment is limited by our understanding of the mechanisms underlying their effects on cancer biology. Local anesthetics have been proved to preferentially inhibit cancer stem cells which imply that local anesthetics target colon cancer stem cell to suppress cancer progressing. Here this paper will review and propose several potential studies, including using colon cancer cell lines and animal models to test the effect of local anesthetics on population, viability, and migration of colon cancer stem-like cell, and screen and search for potential molecular targets underlying these effects.
\end{abstract}

\section{Mini Review}

Colorectal cancer is the third most prevalent cancer and the second most frequent cause of cancer-related death in the world., Surgical resection of the primary tumor is the central aspect of the current multiple modes of treatment and has been associated with better prognosis. However, metastasis is the dominant cause of death in patients with colon cancer. On top of cell migration, chemokines have been implicated in additional aspects of malignant transformation, such as proliferation, survival, and angiogenesis. The process of surgery, including anesthetic regimens, has increasingly been recognized to affect colon cancer recurrence and metastasis. Retrospective clinical studies have suggested that the use of regional anesthesia leads to improved patient outcomes., Laboratory studies have reported that colon cancer cells are inhibited by some local anesthetics. However, the application of local anesthetics in colon cancer treatment is limited by our understanding of the mechanisms underlying their effects on cancer biology. Emerging evidence has indicated a subpopulation of stem-like cells within tumors, known as CSCs, which contribute to cancer treatment failure and cancer relapse. ,
The cancer stem cell hypothesis is rising to be an attractive cellular mechanism that proposes a hierarchical organization within the colon tumor bulk and justifies the functional heterogeneity of solid tumors responsible for the aggressive nature of the malignancy and therapeutic refractoriness [1-10].

Amide-linked local anesthetics, lidocaine, ropivacaine, and bupivacaine, have been proved to preferentially inhibit colony formation and self-renewal of cancer stem cells. A recent study also showed that local anesthetics inhibit colon cancer not through inducing apoptosis or damaging the cell (cytotoxicity) but through arresting cell proliferation cycle. These conclusions imply that local anesthetics target colon cancer stem-like cell to suppress cancer progressing. We aim to determine the effect of local anesthetics on population, viability, and migration of colon cancer stem-like cell, and screen and search for potential molecular targets underlying these effects. The information about their potency and efficacy against colon cancer stem-like cells and the potential targets would help explain the mechanism of effect of regional anesthesia on colon cancer [11-24]. 


\section{Hypothesis and Potential Research in This Field}

a) Here, based on previous knowledge and studies, this review proposed a hypothesis that local anesthetics target colon cancer stem cell to suppress cancer progressing. There are several aspects that can be explored: To explore the effects of local anesthetics on colon cancer stem-like cells.

b) To explore the effects of local anesthetics on colon cancer stem-like cell in a murine cancer model.

c) To explore the effects of local anesthetics on the stem-like cell population in clinical colon cancer tissue.

d) To explore the potential stemness-correlate molecular mechanism underlying the effect.

\section{Potential Available Methods and Strategies}

This review proposed several potential available Methods and strategies.

\section{The Sorting of Stem-Like Cell Subpopulation}

Immuno-magnetic cell sorting of stem cell subpopulation will be used. Human colorectal carcinoma cell lines HCT116, SW480 and HT29 will be exposed to FITC-conjugated anti-CD44, anti-ALDH, and anti-LGR5 antibody, and further labeled with dextran-coated magnetic nanoparticles using bispecific Tetrameric Antibody Complexes (TAC). These cells will be subjected to immunomagnetic cell separation and CD44+, ALDH+, and LGR5+ cells will be identified as cancer stem cells. Sphere culture will also be used as another method to extract cancer stem-like cells. Cells will be cultured in ultralow attachment plates and the sphere passage cells will be identified as cancer stem cells.

\section{The Effects of Local Anesthetics on Colon Cancer Stem- Like Cell}

The colon cancer cell lines and cancer stem-like cell line (sorted cancer cell line derived cells) will be used to test the effects of local amide-linked local anesthetics (lidocaine, ropivacaine, and bupivacaine). Local anesthetics have a wide range of uses in clinical practice and their plasma concentrations can vary widely and the peak plasma concentrations ranging between 1 and $3 \mu \mathrm{M}$., Clinically relevant concentrations will be used to treat the cell. 1, 2, 4, 12, 24, 48 , and $72 \mathrm{~h}$ will be set as treatment time. Cell viability, migration, invasion, and adhesion of cell lines will be determined using MTT, Cell Cytotoxicity Assay Kit, apoptosis ELISA Kit, Flow Cytometry, wound healing, trans well, matrices assay, colony-forming assay, trypsin detaching assay, etc. Self-renewal ability of cell lines will be determined using serial replating assays. The population of cancer stem-like cells in HCT116 and HT29 will be determined by cancer stem cell markers stain using the Immunohistochemistry and Flow Cytometry method.

\section{The Effects of Local Anesthetics on Colon Cancer Stem- Like Cell in A Murine Colon Cancer Model}

Immunodeficient NSG mouse model with implantation of green fluorescent protein-labeled colon cancer cells (HT29) and cancer stem-like cells (HT29 sorted by the method described previously) will be used. In the long-term treatment group, the primary tumor will be treated with clinically relevant concentration local perfusion of local anesthetics per day, while in the short-term treatment group, the animals will only be treated during the surgery. Tumor development will be monitored through longitudinal magnetic resonance imaging-based morphometric analysis and survival. Established serum markers of tumor spread will be measured serially and circulating tumor cells will be detected via fluorescence measurements. The primary tumor will be excised and collected after four weeks and the primary recurrent tumor and metastatic tissue (lung, brain, bone, etc.) will be collected. The population of cancer stem-like cell and in tumor and metastatic tissue will be determined by markers stain using Immunohistochemistry method.

\section{The Effects of Local Anesthetics on The Stem-Like Cell Population in Clinical Colon Cancer Tissue}

To determine whether local anesthetics can decrease colon cancer stem-like cell population in cancer tissue from patients, the colon cancer tissues with complete clinical pathological data will be collected from patients with and without lidocaine treatment. The Paraffin-embedded tissue microarray was constructed and the cancer stem-like cell population in cancer tissue will be determined by cancer stem cell markers, (CD44+, CD24-/low, ALDH+ and LGR5+) stain using the Immunohistochemistry method.

\section{The Effect of Local Anesthetics on Stemness-Correlate Molecular Features}

The colon cancer cell lines and cancer stem-like cell line will be treated with local anesthetics. Total protein and total RNA of cells will be extracted, and proteomic analysis and gene array method will be used to screen altered protein and gene expression. QPCR, Immunofluorescence stain, Western blot, and Immunohistochemistry methods will be used to confirm the results. Gene-knock-out method will be used to test the potential targets of local anesthetics involved in stemness-correlate signal.

\section{Conclusion}

This study hopes to emerge with a detailed clarification of the effect of local anesthetics on population, viability, and migration of colon cancer stem-like cell and specific molecular targets underlying these effects, and in turn, deepen our understanding of local anesthetics in colon cancer treatments. Here, this review proposed that 
a) Colon cancer stem-like cells will be identified and sorted out of colon cancer cell lines.

b) Local anesthetics can suppress viability, migration, invasion, and adhesion more in colon cancer stem-like cell line than in colon cancer cell lines. The population of colon cancer stem-like cell in colon cancer cell lines will decrease after treating with local anesthetics.

c) The population of colon cancer stem-like cell in tumor and metastatic tissue will decrease and cancer progression will be suppressed in local anesthetics treatment groups.

d) The tumor tissue from patients with local anesthetics treatment has less cancer stem-like cells.

e) Several targets of Local anesthetics will be identified. Advantageous molecular targets of stemness will be suppressed and disadvantageous targets of stemness will be promoted in colon cancer cells. Knocking out/down these targets can block the effects of the local anesthetics.

\section{Acknowledgment}

The author thank the support of Zongxiong Liu, Weifen Chen, and Yaqi Yang.

\section{Conflict of Interest}

The author claims that there is no conflict of interest.

\section{References}

1. Ferlay J, Soerjomataram I, Dikshit R, Eser S, Mathers C, et al. (2015) Cancer incidence and mortality worldwide: sources methods and major patterns in GLOBOCAN 2012 International journal of cancer 136(5): E359-E386.

2. Usher Smith JA, Walter FM, Emery JD, Win AK, Griffin SJ (2016) Risk prediction models for colorectal cancer: a systematic review. Cancer prevention research 9(1): 13-26.

3. Vatandoust S, Price TJ, Karapetis CS (2015) Colorectal cancer: Metastases to a single organ World. Journal of Hastroenterology 21(41): 11767.

4. Singh R, Lilladr JW, Singh S (2011) Chemokines: key players in cancer progression and metastasis. Frontiers in bioscience (Scholar edition) 3: 1569.

5. O'Leary DP, O'leary E, Foley N, Cotter TG, Wang JH (2016) Effects of surgery on the cancer stem cell niche. European Journal of Surgical Oncology (EJSO) 42(3): 319-325.

6. Royds J, Khan AH, Buggy DJ (2016) An update on existing ongoing prospective trials evaluating the effect of anesthetic and analgesic techniques during primary Cancer surgery on Cancer recurrence or metastasis. International anesthesiology clinics 54(4): e76-e83.

7. Call TR, Pace NL, Thorup DB, Maxfield D, Chortkoff B, et al. (2015) Factors associated with improved survival after resection of pancreatic Adenocarcinoma. A multivariable model Anesthesiology. The Journal of the American Society of Anesthesiologists 122(2): 317-324.
8. Tianci Li, Lin Chen (2018) Both Bupivacaine and Levobupivacaine inhibit colon cancer cell growth but not melanoma cells in vitro. Journal of Anesthesia.

9. Yu Z, Pestell TG, Lisanti MP, Pestell RG (2012) Cancer stem cells. The International Journal of Biochemistry \& Cell Biology Volume 44(12): 2144-2151.

10. Dragu DL, Necula LG, Bleotu C, Diaconu CC, Chivu Economescu M (2015) Therapies targeting cancer stem cells: current trends and future challenges. World journal of stem cells 7(9): 1185-1201.

11. Boman BM, Huang E (2008) Human colon cancer stem cells: a new paradigm in gastrointestinal oncology. Journal of Clinical Oncology 26(17): 2828-2838.

12. Ricci Vitiani L, Fabrizi E, Palio E (2009) De Maria R Colon cancer stem cells. J Mol Med 87: 1097-1104.

13. Ni J, Xie T, Xiao M, Xiang W, Wang L (2018) Amide-linked local anesthetics preferentially target leukemia stem cell through inhibition of Wnt/ $\beta$ catenin. Biochemical and Biophysical Research Communications 503(2): 956-962.

14. Bundscherer AC, Malsy M, Bitzinger DI, Wiese CH, Gruber MA, et al. (2017) Effects of lidocaine on HT-29 and SW480 colon cancer cells in vitro. Anticancer research 37(4): 1941-1945.

15. Patel S, Waghela B, Shah K, Vaidya F, Mirza S (2018) Silibinin A Natural Blend In Polytherapy Formulation For Targeting Cd44v6 Expressing Colon Cancer Stem Cells. Scientific reports 8(1): 16985.

16. Cao L, Zhou Y, Zhai B, Liao J, Xu W, et al. (2011) Sphere-forming cell subpopulations with cancer stem cell properties in human hepatoma cell lines. BMC gastroenterology 11(1): 71

17. Weiswald LB Bellet D, Dangles Marie V (2015) Spherical cancer models in tumor biology. Neoplasia 17(1): 1-15.

18. Yasumura R, Kobayashi Y, Ochiai R (2016) A comparison of plasma levobupivacaine concentrations following transversus abdominis plane block. and rectus sheath block. Anaesthesia 71(5): 544-549.

19. Trabelsi B, Charfi R, Bennasr L, Marzouk SB, Eljebari H, et al. (2017) Pharmacokinetics of bupivacaine after bilateral ultrasound-guided transversus abdominis plane block following cesarean delivery under spinal anesthesia. International journal of obstetric anesthesia 32: 1720.

20. Huntly BJ, Shigematsu H, Deguchi K, Lee BH, Mizuno S, et al. (2004) MOZ-TIF2 but not BCR-ABL confers properties of leukemic stem cells to committed murine hematopoietic progenitors. Cancer cell 6(6): 587596.

21. Xu H, Li J, Chen H, Ghishan FK (2019) NHE8 Deficiency Promotes ColitisAssociated Cancer in Mice via Expansion of Lgr5-Expressing Cells. Cellular and molecular gastroenterology and hepatology 7(1): 19-31.

22. Al-Hajj M, Wicha MS, Benito-Hernandez A, Morrison SJ, Clarke MF (2003) Prospective identification of tumorigenic breast cancer cells. Proceedings of the National Academy of Sciences 100(7): 3983-3988.

23. Ginestier C, Hur MH, Charafe-Jauffret E, Monville F, Dutcher J, et al. (2007) ALDH1 is a marker of normal and malignant human mammary stem cells and a predictor of poor clinical outcome. Cell stem cell 1(5): 555-567.

24. Shimokawa M, Ohta Y, Nishikori S, Matano M, Takano A, et al. (2017) Visualization and targeting of LGR5+ human colon cancer stem cells. Nature 545(7653): 187. 


\section{ISSN: 2574-1241}

DOI: 10.26717/BJSTR.2020.25.004166

Hengrui Liu. Biomed J Sci \& Tech Res

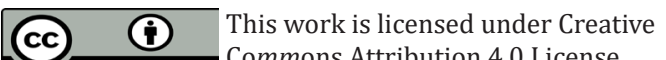

Submission Link: https://biomedres.us/submit-manuscript.php

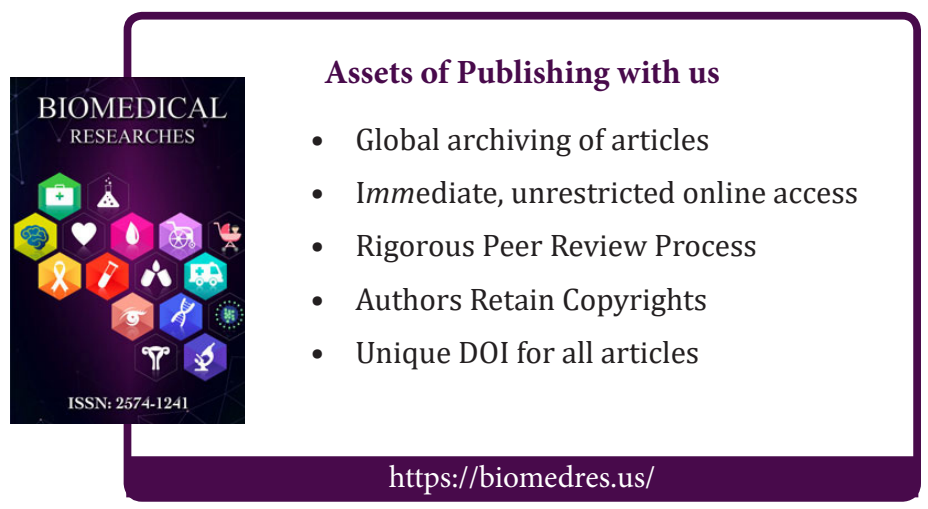

